\title{
A NOTE ON THE WHITEHEAD TORSION OF A BUNDLE MODULO A SUBBUNDLE
}

\author{
DOUGLAS R. ANDERSON
}

\begin{abstract}
The purpose of this note is to show how the sum theorem for Whitehead torsion due to K. W. Kwun and R. H. Szczarba, and generalized by L. C. Siebenmann, may be applied to compute the Whitehead torsion of the total space pair of a bundle pair in terms of the Whitehead torsion of the fiber pair and the Euler characteristic of the base.
\end{abstract}

Let $p: E \rightarrow B$ be a $P L$ fiber bundle with fiber $F$ and suppose $p^{\prime}: E^{\prime} \rightarrow B$ is a $P L$ subbundle with fiber $F^{\prime}$. If the inclusion $F^{\prime} \subset F$ is a homotopy equivalence, a theorem of Dold [1, Theorem 6.3] implies that the inclusion $E \subset E^{\prime}$ is also a homotopy equivalence. It is the object of this note to answer the natural question" "How are the Whitehead torsions $\tau\left(F, F^{\prime}\right) \in$ Wh $\pi_{1}(F)$ and $\tau\left(E, E^{\prime}\right) \in \mathrm{Wh} \pi_{1}(E)$ related ?" Specifically we prove the

ThEOREM. Let $B$ and $F$ be connected. Then

$$
\tau\left(E, E^{\prime}\right)=\chi(B) j_{*} \tau\left(F, F^{\prime}\right)
$$

where $\chi(B)$ is the Euler characteristic of $B$ and $j_{*}:$ Wh $\pi_{1}(F) \rightarrow$ Wh $\pi_{1}(E)$ is induced by the inclusion $j: F \rightarrow E$.

To set the context precisely, we recall that a $P L$ bundle pair with fiber $\left(F, F^{\prime}\right)$ is a $P L$ map $p:\left(E, E^{\prime}\right) \rightarrow(B, B)$, denoted simply by $B$ in the sequel, such that for some triangulation $K$ of $B$ and each simplex $\sigma \in K$, there is a $P L$ homeomorphism $h_{\sigma}:\left(\sigma \times F, \sigma \times F^{\prime}\right) \rightarrow\left(p^{-1}(\sigma), p^{-1}(\sigma) \cap E^{\prime}\right)$ such that $p h_{\sigma}=p_{1}$ where $p_{1}:\left(\sigma \times F, \sigma \times F^{\prime}\right) \rightarrow \sigma$ is projection on the first factor. A $P L$ bundle $p^{\prime}: E^{\prime} \rightarrow B$ with fiber $F^{\prime}$ is a subbundle of the $P L$ bundle $p: E \rightarrow B$ with fiber $F$ if $E^{\prime} \subset E, p^{\prime}=p \mid E^{\prime}$, and $p:\left(E, E^{\prime}\right) \rightarrow B$ is a $P L$ bundle pair with fiber $\left(F, F^{\prime}\right)$. In this event for each $x \in B$ we denote the pair $\left(p^{-1}(x), p^{\prime-1}(x)\right)=\left(p^{-1}(x), p^{-1}(x) \cap E^{\prime}\right)$ by $\left(F_{x}, F_{x}^{\prime}\right)$ and the inclusion $\left(F_{x}, F_{x}^{\prime}\right) \subset\left(E, E^{\prime}\right)$ by $j_{x}$.

Throughout the remainder of this note we work in the category of compact $P L$ spaces and $P L$ maps.

Received by the editors June 1, 1971.

AMS 1970 subject classifications. Primary 57C10; Secondary 57C50.

Key words and phrases. Whitehead torsion, bundle pair.

${ }^{1}$ Raised by $\mathrm{K}$. Millett in conversation with the author.

(c) American Mathematical Society 1972 
The only technical concern in the proof of the theorem is the handling of base points. This concern is obviated by the

LEMMA. Let $p:\left(E, E^{\prime}\right) \rightarrow B$ be a bundle pair with connected base and fiber $\left(F, F^{\prime}\right)$. Suppose $F$ is connected and the inclusion $F^{\prime} \subset F$ is a homotopy equivalence. Then for any points $b, c \in B, j_{b *} \tau\left(F_{b}, F_{b}^{\prime}\right)=j_{c *} \tau\left(F_{c}, F_{c}^{\prime}\right)$ where $\tau\left(F_{x}, F_{x}^{\prime}\right)$ denotes the Whitehead torsion of $\left(F_{x}, F_{x}^{\prime}\right), x=b, c$.

Proof. Let $g: I \rightarrow B$ be a path with $g(0)=b$ and $g(1)=c$. Then there is a bundle map $G:\left(I \times F, I \times F^{\prime}\right) \rightarrow\left(E, E^{\prime}\right)$ covering $g$. Letting $g_{i}=G \mid i \times F$ for $i=0,1$, and $\sigma=G \mid I \times x_{0}$ where $x_{0} \in F$ is a base point, we obtain a commutative diagram

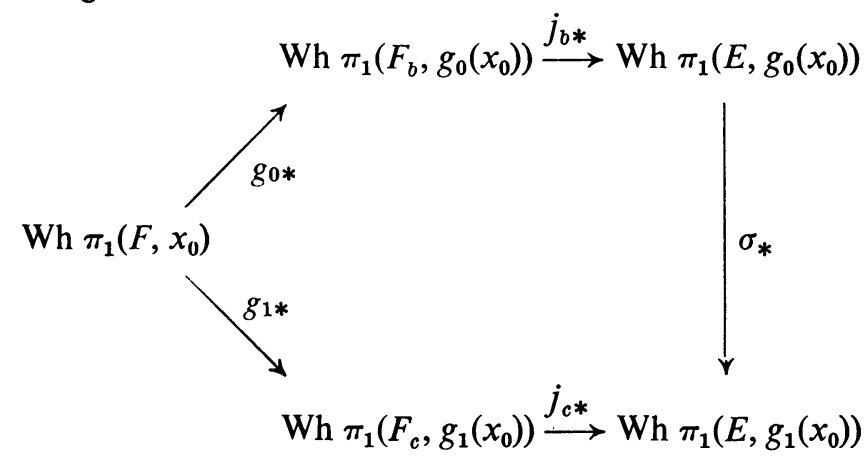

where $\sigma_{*}$ is induced by the "change of base point" homomorphism $\sigma_{\#}: \pi_{1}\left(E, g_{0}\left(x_{0}\right)\right) \rightarrow \pi_{1}\left(E, g_{1}\left(x_{0}\right)\right)$. Since $g_{0}:\left(F, F^{\prime}\right) \rightarrow\left(F_{b}, F_{b}^{\prime}\right)$ is a $P L$ homeomorphism $g_{0 *} \tau\left(F, F^{\prime}\right)=\tau\left(F_{b}, F_{b}^{\prime}\right)$. Similarly $g_{1 *} \tau\left(F, F^{\prime}\right)=\tau\left(F_{c}, F_{c}^{\prime}\right)$. The lemma now follows from the commutativity of the diagram since $\sigma_{*}$ is the canonical isomorphism used in defining Wh $\pi_{1}(E)$ as an abstract group without reference to base points.

Since $j_{b *} \tau\left(F_{b}, F_{b}^{\prime}\right)$ is independent of $b \in B$, we will denote this element of Wh $\pi_{1}(E)$ unambiguously by $j_{*} \tau\left(F, F^{\prime}\right)$ and will suppress any further reference to the choice of $b$.

Proof of The Theorem. Let $K$ be a triangulation of $B, K_{0} \subset K$ be a maximal tree, and $\sigma_{1}, \sigma_{2}, \cdots, \sigma_{m}$ be the simplices of $K-K_{0}$ ordered in such a way that if $\operatorname{dim} \sigma_{i}<\operatorname{dim} \sigma_{j}$, then $i<j$. Note that $\operatorname{dim} \sigma_{i} \geqq 1$ for all $i$. For $n>0$, let $K_{n}=K_{0} \cup \bigcup_{i=1}^{n} \sigma_{i}$ and set $\left(E_{n}, E_{n}^{\prime}\right)=\left(p^{-1}\left(K_{n}\right), p^{\prime-1}\left(K_{n}\right)\right)$. The proof proceeds by induction on $n$.

If $n=0$, there is an equivalence of bundle pairs $h:\left(K_{0} \times F, K_{0} \times F^{\prime}\right) \rightarrow$ $\left(E_{0}, E_{0}^{\prime}\right)$ since $K_{0}$ is collapsible. Hence

$$
\begin{aligned}
\tau\left(E_{0}, E_{0}^{\prime}\right) & =h_{*} \tau\left(K_{0} \times F, K_{0} \times F^{\prime}\right) \\
& =\chi\left(K_{0}\right) h_{*} k_{*} \tau\left(F, F^{\prime}\right) \\
& =\chi_{(}\left(K_{0}\right) j_{*} \tau\left(F, F^{\prime}\right)
\end{aligned}
$$


by the Product Theorem [2] where $k:\left(F, F^{\prime}\right) \rightarrow\left(K_{0} \times F, K_{0} \times F^{\prime}\right)$ is the inclusion of the fiber pair.

Suppose the theorem holds for $\left(E_{n-1}, E_{n-1}^{\prime}\right)$. Since $E_{n}=E_{n-1} \cup p^{-1}\left(\sigma_{n}\right)$, $E_{n-1} \cap p^{-1}\left(\rho_{n}\right)=p^{-1}\left(\dot{\sigma}_{n}\right)$, and $E_{n}^{\prime}$ has a similar decomposition,

$$
\begin{aligned}
\tau\left(E_{n}, E_{n}^{\prime}\right)= & k_{1 *} \tau\left(E_{n-1}, E_{n-1}^{\prime}\right)+k_{2 *} \tau\left(p^{-1}\left(\sigma_{n}\right), p^{\prime-1}\left(\sigma_{n}\right)\right) \\
& -k_{3 *} \tau\left(p^{-1}\left(\dot{\sigma}_{n}\right), p^{\prime-1}\left(\dot{\sigma}_{n}\right)\right)
\end{aligned}
$$

by the Sum Theorem [2] and [3] where $k_{1 *}, k_{2 *}$, and $k_{3 *}$ are induced by the obvious inclusions into $\left(E_{n}, E_{n}^{\prime}\right)$. By the induction hypothesis,

$$
k_{1 *} \tau\left(E_{n-1}, E_{n-1}^{\prime}\right)=\chi\left(K_{n-1}\right) j_{*} \tau\left(F, F^{\prime}\right) .
$$

Also since $\sigma_{n}$ is contractible, there is an equivalence of bundle pairs $h:\left(\sigma_{n} \times F, \sigma_{n} \times F^{\prime}\right) \rightarrow\left(p^{-1}\left(\sigma_{n}\right), p^{\prime-1}\left(\sigma_{n}\right)\right)$. Hence by the Product Theorem [2],

$$
\begin{aligned}
k_{2 *} \tau\left(p^{-1}\left(\sigma_{n}\right), p^{\prime-1}\left(\sigma_{n}\right)\right) & =k_{2 *} h_{*} \tau\left(\sigma_{n} \times F, \sigma_{n} \times F^{\prime}\right) \\
& =\chi\left(\sigma_{n}\right) k_{2 *} h_{*} k_{*} \tau\left(F, F^{\prime}\right) \\
& =\chi\left(\sigma_{n}\right) j_{*} \tau\left(F, F^{\prime}\right)
\end{aligned}
$$

where $k:\left(F, F^{\prime}\right) \rightarrow\left(\sigma_{n} \times F, \sigma_{n} \times F^{\prime}\right)$ is the inclusion of the fiber pair Similarly $k_{3 *} \tau\left(p^{-1}\left(\dot{\sigma}_{n}\right), p^{\prime-1}\left(\sigma_{n}\right)\right)=\chi\left(\dot{\sigma}_{n}\right) j_{*} \tau\left(F, F^{\prime}\right)$ at least if $\operatorname{dim} \sigma_{n}>1$. (If $\operatorname{dim} \sigma_{n}=1$, this follows from the lemma.) Hence

$$
\tau\left(E_{n}, E_{n}^{\prime}\right)=\left[\chi\left(K_{n-1}\right)+\chi\left(\sigma_{n}\right)-\chi\left(\dot{\sigma}_{n}\right)\right] j_{*} \tau\left(F, F^{\prime}\right)=\chi\left(K_{n}\right) j_{*} \tau\left(F, F^{\prime}\right)
$$

and the theorem follows by induction.

\section{BIBLIOGRAPHY}

1. A. Dold, Partitions of unity in the theory of fibrations, Ann. of Math. (2) 78 (1963), 223-255. MR 27 \#5264.

2. K. W. Kwun and R. H. Szczarba, Product and sum theorems for Whitehead torsion, Ann. of Math. (2) 82 (1965), 183-190. MR 32 \#454.

3. L. C. Siebenmann, The obstruction to finding a boundary for an open manifold of dimension greater than five, Thesis, Princeton University, Princeton, N.J., 1965.

Department of Mathematics, Syracuse University, Syracuse, New York 13210 